\title{
Alcohol septal ablation: in which patients and why?
}

\author{
Paolo Spirito ${ }^{1}$, Jessica Rossi ${ }^{1}$, Barry J. Maron ${ }^{2}$ \\ ${ }^{1}$ Hypertrophic Cardiomyopathy Center, Policlinico di Monza, Monza, Italy; ${ }^{2}$ HCM Institute, Division of Cardiology, Tufts Medical Center, Boston, \\ MA, USA \\ Correspondence to: Paolo Spirito, MD. Hypertrophic Cardiomyopathy Center, Policlinico di Monza, Monza, Italy. \\ Email: paolo.spirito@policlinicodimonza.it.
}

\begin{abstract}
At present, surgical septal myectomy is regarded as the "gold standard" treatment for most patients with obstructive hypertrophic cardiomyopathy (HCM) and drug-refractory symptoms. However, the best results are obtained by those surgeons who have extensive experience with this operation at a small number of referral centers. In the mid-1990s, percutaneous alcohol septal ablation was introduced as an alternative to myectomy to reduce $\mathrm{LV}$ outflow gradient and heart failure symptoms in patients with obstructive HCM. However, certain features of alcohol ablation limit its applicability to carefully selected patients. Because this procedure involves the injection of $1-4 \mathrm{~mL}$ of $96 \%$ ethanol into a septal perforator of the left anterior coronary artery to produce a myocardial infarction (with septal thinning, outflow tract widening and gradient reduction), alcohol ablation is limited by the size and distribution of the septal perforator branches. In addition, rich blood supply from other septal branches not occluded by the balloon and from the posterior descending coronary artery may restrict myocardial ischemia to portions of the septum that do not contribute to outflow obstruction. Septal hypertrophy may be either mild or particularly marked, and abnormalities of mitral valve apparatus may also play a major role in outflow obstruction. When such features are present, alcohol-induced septal thinning is unlikely to significantly reduce the gradient. In addition, persisting uncertainties regarding the risk for ventricular tachyarrhythmias after alcohol ablation suggest this procedure should be limited to patients of advanced age, patients at unacceptable operative risk due to comorbidities, or those with strong aversion to surgery. Further progress in the treatment for patients with obstructive HCM and severe refractory symptoms will come from assuring proper patient selection for alcohol septal ablation, as well as increasing the number of surgeons and centers experienced in performing septal myectomy.
\end{abstract}

Keywords: Septal ablation; hypertrophic cardiomyopathy (HCM)

Submitted Feb 13, 2017. Accepted for publication Apr 04, 2017.

doi: 10.21037/acs.2017.05.09

View this article at: http://dx.doi.org/10.21037/acs.2017.05.09

In patients with hypertrophic cardiomyopathy (HCM), left ventricular (LV) outflow obstruction with a pressure gradient between the LV outflow tract and aorta is caused by systolic anterior motion of the mitral valve against the septum (1), and is identified at rest or with physiologic exercise in about $70 \%$ of patients evaluated at tertiary referral centers (2). Obstruction to LV outflow obstruction is associated with mitral valve regurgitation, disabling heart failure symptoms and increased cardiovascular mortality $(3,4)$. For almost 50 years, transaortic septal myectomy has represented the standard surgical approach for the treatment of patients with obstructive HCM and severe symptoms refractory to medical treatment (1,5-7).

In the mid-1990s, percutaneous alcohol septal ablation was introduced as an alternative to surgical myectomy to reduce the $\mathrm{LV}$ outflow gradient and heart failure symptoms in patients with obstructive HCM (8). This catheterbased procedure, made attractive by its less invasive nature, was rapidly and widely adopted in catheterization laboratories world-wide, most notably in Europe, where several time-honored surgical myectomy programs were replaced by alcohol ablation within just a few years (9). 
At present, only a small number of European HCM surgical referral centers continue to perform a high volume of myectomy operations, mainly in Italy, the Netherlands and Belgium (10-13). Although alcohol septal ablation was also adopted by interventional cardiologists in the United States, this procedure seems to have had the unexpected and paradoxical effect of increasing the number of surgical myectomies, possibly by enhancing awareness of the importance of LV outflow obstruction on the clinical course of HCM patients (14). A longer tradition for surgical treatment of HCM in North America and a culture in which patients are more prepared than their European counterparts to travel long distances to obtain highly specialized treatment, may have also contributed.

For this issue of the Annals of Cardiothoracic Surgery, dedicated to obstructive HCM, we provide a perspective on alcohol septal ablation, and in particular, a clarification regarding the appropriate selection of candidates for this percutaneous treatment, as well as the justifications for such clinical decisions. This article compares, in a balanced fashion, the relative advantages and limitations of surgical myectomy $v s$. alcohol ablation.

\section{Surgical septal myectomy}

During the last 20 years, a decrease in operative mortality due to improved surgical techniques and myocardial protection has reduced the hospital mortality for isolated septal myectomy to $<1 \%$ at HCM Centers of Excellence $(5,7,10)$. In addition, optimal hemodynamic results and sustained clinical improvement to New York Heart Association class I or II have been documented in $>90 \%$ of patients over long-term follow-up $(5,7,10)$. The exceedingly low operative mortality and major long-term benefits of transaortic surgical myectomy (with extended survival equivalent to patients with non-obstructive $\mathrm{HCM}$ and similar to the age and gender matched general population) is now well documented for high volume centers $(1,5,7,10)$.

However, septal myectomy is a highly specialized and relatively uncommon cardiac operation. As there is a direct relation between volume of myectomies performed and surgical outcomes, optimal results cannot be expected from surgeons with a low-volume exposure to symptomatic patients with obstructive HCM $(1,15,16)$. Therefore, the best results have been obtained by those few surgeons in the world with extensive myectomy experience and a small number of referral centers in North America and Europe
(5,7,11,17-20). This represents an important obstacle to bringing septal myectomy to the HCM population. While there is no easy solution to this problem, it is important to promote septal myectomy, encourage other cardiac surgeons to gain experience with this operation and to develop additional HCM referral centers that include surgical treatment (20). Furthermore, similar programs should be implemented in developing countries that (at present) cannot offer the option of surgical myectomy to patients with obstructive HCM. The recent development of the first Indian surgical referral center for HCM in New Delhi demonstrates that motivation along with successful planning and implementation can create such a programeven under difficult circumstances (21).

\section{Alcohol septal ablation}

Alcohol septal ablation is a widely available percutaneous, catheter-based technique that can be performed in virtually any laboratory and avoids most of the inconvenience associated with surgery. In particular, alcohol ablation requires a shorter hospital admission and recovery time than surgery and is associated with less postoperative disability. In the last decade, a large body of literature (including a number of meta-analyses) has reported the results of this procedure (13,22-26). Alcohol septal ablation can often relieve obstruction and improve symptoms (at least during an intermediate period of follow-up) when performed in properly selected patients by interventional cardiovascular specialists with extensive experience with this procedure $(13,22,24,25)$. However, a note of caution should be raised regarding limitations of the literature on alcohol ablation. The same features that make this technique widely available (including the possibility of being performed by a majority of interventional cardiologists without prior specialized training) may have generated a situation where alcohol ablation is performed in many laboratories with very limited experience. Unfavorable results, including severe complications and death may not be reported in the literature.

However, even when performed by interventional cardiologists with extensive experience with alcohol septal ablation, certain features of this technique (along with the complex morphology and pathophysiology of HCM) limit the applicability of alcohol ablation to carefully selected patients. Therefore, at present, the central question is: who are the proper candidates? The recommendations of the US/Canada (ACC/AHA) guidelines for the treatment 
of HCM, published in 2011, provide an answer to this question: "Surgical septal myectomy, when performed by experienced operators, ... is the first consideration for the majority of eligible patients with HCM", and, "when surgery is contraindicated, or the risk is considered unacceptable because of serious co-morbidity or advanced age, alcohol septal ablation, when performed in experienced centers, can be beneficial in eligible adult patients with HCM with LV outflow obstruction and severe drug refractory symptoms" (1). These guidelines were assembled by a panel of experts that included cardiologists and cardiac surgeons with extensive experience in treatment of patients with HCM and interventional cardiologists with expertise in alcohol septal ablation. However, such recommendations could not be based on evidence derived from prospective comparisons of surgical myectomy and alcohol septal ablation results, as HCM is a relatively uncommon disease not amenable to randomized trials (27). The conclusions of this expert panel thus reflect practical considerations derived from available short and long-term results of surgical septal myectomy, as well as data and experience for alcohol septal ablation over a much shorter time period.

\section{Technical issues}

Alcohol septal ablation involves the injection of 1 to $4 \mathrm{~mL}$ of $96 \%$ ethanol into a proximal perforator branch of the left anterior descending coronary artery to produce a myocardial infarction of the basal septum with a decrease in septal contractility. The septal scarring and thinning results in widening of the outflow tract and decrease or abolition of the LV outflow gradient $(1,8)$. However, injecting ethanol into a proximal branch of the left anterior descending artery does not guarantee the distribution of alcohol (and myocardial infarction) will be confined to the basal septum. This is because the septum is the most vascularized area of the left ventricle and receives blood from all other septal branches not occluded by the balloon, as well as from the posterior descending artery (28-30). These small arteries form an intricate network of anastomoses that may either limit the area of myocardial ischemia to segments of the septum that do not contribute to outflow obstruction or extend ischemia to other areas of the left ventricle with the potential for widespread myocardial damage (31-33). This difficulty in predicting the myocardial distribution of alcohol is also the basis for the $10 \%$ to $15 \%$ rate of complete heart block and permanent pacemaker implantation, as the conduction system is located in the posterior ventricular septum and consequently exposed to damage by the injected ethanol $(1,8,12,22,25,30)$. The risk of such complications is directly related to operator and institutional experience with the alcohol septal ablation procedure.

\section{Morphologic complexity of HCM}

The structural heterogeneity of the LV outflow tract and complex pathophysiology of outflow obstruction in HCM influence the selection of candidates for alcohol ablation. Patients with obstructive HCM often have mitral valve abnormalities, including papillary muscle displacement within the LV cavity, fibrotic and retracted secondary mitral valve chordae and markedly elongated mitral valve leaflets that can contribute to outflow obstruction $(11,17,31-33)$. In such patients, septal myectomy is often associated with mitral valve repair $(7,11,32,34)$. A number of novel valve repair techniques have been developed that reduce the contribution of the mitral valve apparatus to outflow obstruction. Such techniques include mobilization of the papillary muscles, secondary anterior leaflet chordal cutting, and extension or plication of the anterior mitral leaflet $(11,18,19)$. Of note, septal myocardial infarction caused by alcohol cannot abolish the outflow gradient in HCM patients where abnormalities of the mitral valve apparatus play a major role in LV outflow obstruction and in many instances, mitral valve abnormalities would exclude alcohol ablation as a viable treatment option.

\section{Arrhythmogenic potential of alcohol septal ablation}

The concept of causing a destructive myocardial infarction as a form of therapy has been a source of concern since the introduction of alcohol septal ablation in 1995 (35). HCM is a myocardial disease associated with the risk of lifethreating ventricular tachyarrhythmias $(1,36)$. Therefore, a sizable septal scar resulting from alcohol injection could increase that risk, a concern amplified by the principles on which the procedure is based: the larger the amount of alcohol injected, the larger the myocardial infarction and more marked can the decrease in the outflow gradient become (37). However, a larger scar could also be associated with a higher risk of arrhythmias, and small amounts of alcohol may be associated with persistence of the outflow gradient (37).

At present, the magnitude of risk for ventricular tachyarrhythmias after alcohol septal ablation is 


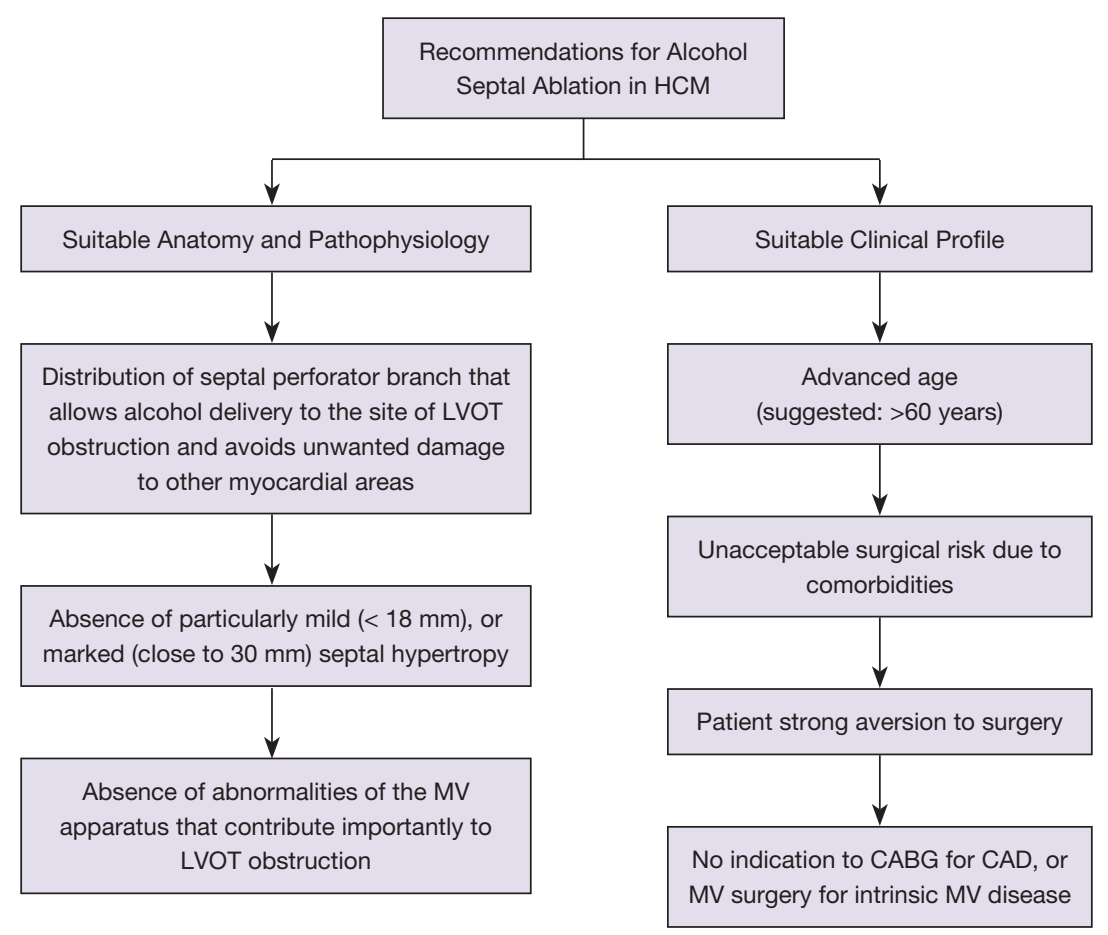

Figure 1 Criteria for selection of alcohol septal ablation as invasive treatment of LV outflow gradient in patients with obstructive HCM and severe drug-refractory heart failure symptoms. CAD, coronary artery disease; CABG, coronary artery by-pass graft; HCM, hypertrophic cardiomyopathy; LVOT, left ventricular outflow; MV, mitral valve.

incompletely resolved and remains a source of some controversy. Results from multicenter studies and metaanalyses comparing incidence of ventricular tachycardia and fibrillation in large cohorts of patients after alcohol septal ablation $v s$. surgical myectomy remain open to contrasting interpretations $(37,38)$. One limitation of such investigations is the shorter period of follow-up and incompleteness of data on appropriate ICD interventions in septal ablation cohorts compared to long-term surgical series (37). Nevertheless, in a recent multicenter European study, appropriate ICD shocks for ventricular tachycardia or fibrillation were 8 -fold more common after septal ablation than surgical myectomy (13). Furthermore, major HCM centers have reported a higher rate of ventricular tachyarrhythmias, cardiac arrest, or appropriate ICD interventions after alcohol septal ablation $(12,38)$, and some centers have prophylactically implanted ICDs for primary prevention after septal ablation (22). In contrast to these recurring concerns regarding arrhythmic risk associated with alcohol septal ablation, available data show that surgical myectomy extends survival with an exceedingly low rate of events, including ventricular tachyarrhythmias and sudden death, during long-term follow-up $(5,7,10)$. Therefore, the issue of mid and long-term arrhythmogenic potential of septal ablation (although not definitively quantified) cannot be ignored and plays an important role in directing patient selection for ablation towards older patients, or those who for different reasons are not candidates for surgical myectomy.

\section{Candidates for alcohol septal ablation}

Due to certain limitations, alcohol septal ablation can only be regarded as an alternative to surgical septal myectomy when performed in selected patients who have suitable coronary artery anatomy, ventricular septum morphology and outflow obstruction pathophysiology (Figure 1). Size and distribution of the anterior coronary artery septal branches must allow delivery of the injected alcohol to the site of outflow tract obstruction, i.e., the area of contact between mitral valve and ventricular septum during systole. Septal hypertrophy should be within a wall thickness range that can be effectively treated with alcohol septal ablation. That is, mild septal hypertrophy $(<18 \mathrm{~mm})$ would 
expose the patient to the risk of extreme wall thinning with potentially unpredictable consequences, while particularly marked septal hypertrophy would not allow sufficient septal thinning to reduce the outflow gradient without introducing a large amount of alcohol and causing an extensive myocardial scar $(1,37)$. Abnormalities of the mitral valve apparatus that play a major role in generating the outflow gradient should be absent (11,31-33).

Alcohol septal ablation also requires selection of patients with a suitable clinical profile (Figure 1). The US/Canada (ACC/AHA) guidelines suggest limiting alcohol septal ablation to patients of advanced age, those at an unacceptably high operative risk as a result of important comorbidities or those with a strong aversion to surgery (1). In particular, the limitation of alcohol ablation to patients of advanced age (arbitrarily $>60$ years) can be justified by the low rate of sudden death events in older HCM patients (39) and their shorter period of potential risk compared to young patients. Finally, patients with concomitant diseases that would independently warrant surgical correction, such as coronary artery bypass grafting or mitral valve repair for ruptured chordae, cannot be candidates for percutaneous alcohol septal ablation (1).

\section{Conclusions}

Surgical septal myectomy remains the "gold standard" (and preferred) treatment for HCM patients with LV outflow obstruction and severe drug-refractory heart failure symptoms. However, the small number of centers with extensive myectomy experience, in the context of a growing number of operative candidates, represents a significant challenge to offering the best invasive treatment for $\mathrm{LV}$ outflow obstruction to a large number of eligible patients. Alcohol septal ablation has evident advantages over surgical myectomy by avoiding the discomfort, anxiety and recovery time associated with surgery and is a potential alternative to septal myectomy when experienced surgeons are simply unavailable. Nevertheless, technical limitations of alcohol ablation represent major obstacles for considering this procedure as applicable to most candidates for invasive treatment, or equivalent to surgical myectomy in terms of long-term improvement in quality of life and extended survival. Further progress in treatment for HCM patients with severe refractory symptoms secondary to LV outflow obstruction will come from increasing the number of surgeons and centers experienced in performing septal myectomy and from assuring proper patient selection for alcohol septal ablation.

\section{Acknowledgements}

None.

\section{Footnote}

Conflicts of Interest: The authors have no conflicts of interest to declare.

\section{References}

1. Gersh BJ, Maron BJ, Bonow RO, et al. 2011 ACCF/AHA guideline for the diagnosis and treatment of hypertrophic cardiomyopathy. Circulation 2011;124:2761-96.

2. Maron MS, Olivotto I, Zenovich AG, et al. Hypertrophic cardiomyopathy is predominantly a disease of left ventricular outflow tract obstruction. Circulation 2006;114:2232-9.

3. Maron MS, Olivotto I, Betocchi S, et al. Effect of left ventricular outflow tract obstruction on clinical outcome in hypertrophic cardiomyopathy. N Engl J Med 2003;348:295-303.

4. Autore $\mathrm{C}$, Bernabò $\mathrm{P}$, Barillà $\mathrm{CS}$, et al. The prognostic importance of left ventricular outflow obstruction in hypertrophic cardiomyopathy varies in relation to the severity of symptoms. J Am Coll Cardiol 2005;45:1076-80.

5. Ommen SR, Maron BJ, Olivotto I, et al. Long-term effects of surgical septal myectomy on survival in patients with obstructive hypertrophic cardiomyopathy. J Am Coll Cardiol 2005;46:470-6.

6. Ball W, Ivanov J, Rakowski H, et al. Long-term survival in patients with resting obstructive hypertrophic cardiomyopathy: comparison of conservative versus invasive treatment. J Am Coll Cardiol 2011;58:2313-21.

7. Desai MY, Bhonsale A, Smedira NG, et al. Predictors of long-term outcomes in symptomatic hypertrophic obstructive cardiomyopathy patients undergoing surgical relief of left ventricular outflow tract obstruction. Circulation 2013;128:209-16.

8. Sigwart U. Non-surgical myocardial reduction for hypertrophic obstructive cardiomyopathy. Lancet 1995;346:211-4.

9. Maron BJ, Yacoub M, Dearani JA. Benefits of surgery for obstructive hypertrophic cardiomyopathy: bring septal myectomy back for European patients. Eur Heart J 2011;32:1055-8. 
10. Iacovoni A, Spirito P, Simon C, et al. A contemporary European experience with surgical septal myectomy in hypertrophic cardiomyopathy. Eur Heart J 2012;33:2080-7.

11. Ferrazzi P, Spirito P, Iacovoni A, et al. Transaortic chordal cutting: mitral valve repair for obstructive hypertrophic cardiomyopathy with mild septal hypertrophy. J Am Coll Cardiol 2015;66:1687-96.

12. ten Cate FJ, Soliman OI, Michels M, et al. Long-term outcome of alcohol septal ablation in patients with obstructive hypertrophic cardiomyopathy: a word of caution. Circ Heart Fail 2010;3:362-9.

13. Vriesendorp PA, Liebregts M, Steggerda RC, et al. Longterm outcomes after medical and invasive treatment in patients with hypertrophic cardiomyopathy. JACC Heart Fail 2014;2:630-6.

14. Maron BJ, Nishimura RA. Revisiting arrhythmic risk after alcohol septal ablation: Is the pendulum finally swinging back to myectomy? JACC Heart Fail 2014;2:637-40.

15. Panaich SS, Badheka AO, Chothani A, et al. Results of ventricular septal myectomy and hypertrophic cardiomyopathy (from Nationwide Inpatient Sample [1998-2010]). Am J Cardiol 2014;114:1390-5.

16. Ommen SR, Nishimura RA. Hypertrophic Cardiomyopathy-One Case per Year?: A Clarion Call to Do What Is Right. JAMA Cardiol 2016;1:333-4.

17. Kaple RK, Murphy RT, Di Paola LM, et al. Mitral valve abnormalities in hypertrophic cardiomyopathy: echocardiographic features and surgical outcomes. Ann Thorac Surg 2008;85:1527-35, 1535.

18. van der Lee C, Kofflard MJ, van Herwerden LA, et al. Sustained improvement after combined anterior mitral leaflet extension and myectomy in hypertrophic obstructive cardiomyopathy. Circulation 2003;108:2088-92.

19. Balaram SK, Ross RE, Sherrid MV, et al. Role of mitral valve plication in the management of hypertrophic cardiomyopathy. Ann Thorac Surg 2012;94:1990-7.

20. Maron BJ, Dearani JA, Maron MS, et al. Why We Need More Septal Myectomy Surgeons: An Emerging Recognition. J Thorac Cardiovasc Surg 2017. In Press.

21. Maron BJ, Spirito P. Importance and feasibility of creating hypertrophic cardiomyopathy centers in developing countries: the experience in India. Am J Cardiol 2015;116:332-4.

22. Cuoco FA, Spencer WH III, Fernandes VL, et al. Implantable cardioverter-defibrillator therapy for primary prevention of sudden death after alcohol septal ablation of hypertrophic cardiomyopathy. J Am Coll Cardiol
2008;52:1718-23.

23. Agarwal S, Tuzcu EM, Desai MY, et al. Updated metaanalysis of septal alcohol ablation versus myectomy for hypertrophic cardiomyopathy. J Am Coll Cardiol 2010;5 5:823-34.

24. Nagueh SF, Groves BM, Schwartz L, et al. Alcohol septal ablation for the treatment of hypertrophic obstructive cardiomyopathy: a multicenter North American registry. J Am Coll Cardiol 2011;58:2322-8.

25. Sorajja P, Ommen SR, Holmes DR Jr, et al. Survival after alcohol septal ablation for obstructive hypertrophic cardiomyopathy. Circulation 2012;126:2374-80.

26. Liebregts M, Vriesendorp PA, Mahmoodi BK, et al. A systematic review and meta-analysis of long-term outcomes after septal reduction therapy in patients with hypertrophic cardiomyopathy. JACC Heart Fail 2015;3:896-905.

27. Olivotto I, Ommen SR, Maron MS, et al. Surgical myectomy versus alcohol septal ablation for obstructive hypertrophic cardiomyopathy: will there ever be a randomized trial? J Am Coll Cardiol 2007;50:831-4.

28. Sorajja P, Nishimura RA. Outcome prediction of septal ablation in patients with hypertrophic cardiomyopathy still a long way to go. JACC Cardiovasc Interv 2011;4:1035-6.

29. van Dockum WG, Beek AM, ten Cate FJ, et al. Early onset and progression of left ventricular remodeling after alcohol septal ablation in hypertrophic obstructive cardiomyopathy. Circulation 2005;111:2503-8.

30. Valeti US, Nishimura RA, Holmes DR, et al. Comparison of surgical septal myectomy and alcohol septal ablation with cardiac magnetic resonance imaging in patients with hypertrophic obstructive cardiomyopathy. J Am Coll Cardiol 2007;49:350-7.

31. Levine RA, Vlahakes GJ, Lefebvre X, et al. Papillary muscle displacement causes systolic anterior motion of the mitral valve: experimental validation and insights into the mechanism of subaortic obstruction. Circulation 1995;91:1189-95.

32. Patel P, Dhillon A, Popovic ZB, et al. Left ventricular outflow obstruction in hypertrophic cardiomyopathy patients without severe septal hypertrophy: implications of mitral valve and papillary muscle abnormalities assessed using cardiac magnetic resonance and echocardiography. Circ Cardiovasc Imaging 2015;8:e003132.

33. Sherrid MV, Balaram S, Kim B, et al. The mitral valve in obstructive hypertrophic cardiomiopathy. J Am Coll Cardiol 2016;67:1846-58.

34. Hong JH, Schaff HV, Nishimura RA, et al. Mitral regurgitation in patients with hypertrophic obstructive 
cardiomyopathy: Implications for concomitant valve procedures. J Am Coll Cardiol 2016; 68:1497-504.

35. Spirito P, Maron BJ. Perspectives on the role of new treatment strategies in hypertrophic obstructive cardiomyopathy. J Am Coll Cardiol 1999;33:1071-5.

36. Maron BJ, Spirito P, Shen WK, et al. Implantable cardioverter-defibrillators and prevention of sudden cardiac death in hypertrophic cardiomyopathy. JAMA 2007;298:405-12.

37. Nishimura RA, Ommen SR. Septal reduction therapy

Cite this article as: Spirito P, Rossi J, Maron BJ. Alcohol septal ablation: in which patients and why? Ann Cardiothorac Surg 2017;6(4):369-375. doi: 10.21037/acs.2017.05.09 for obstructive hypertrophic cardiomyopathy and sudden death. What statistics cannot tell you. Circ Cardiovasc Interv 2010;3:91-3.

38. Noseworthy PA, Rosenberg MA, Fifer MA. Ventricular arrhythmia following alcohol septal ablation for obstructive hypertrophic cardiomyopathy. Am J Cardiol 2009;104:128-32.

39. Maron BJ, Rowin EJ, Casey SA, et al. Risk stratification and outcome of patients with hypertrophic cardiomyopathy over 60 years of age. Circulation 2013;127:585-93. 